\title{
Identification of an Arabidopsis Nodulin-Related Protein in Heat Stress
}

\author{
Qiantang $\mathrm{Fu}^{1,2}$, Shujia $\mathrm{Li}^{1}$, and Diqiu $\mathrm{Yu}^{1, *}$
}

\begin{abstract}
We identified a Nodulin-related protein 1 (NRP1) encoded by At2g03440, which was previously reported to be RPS2 interacting protein in yeast-two-hybrid assay. Northern blotting showed that AtNRP1 expression was suppressed by heat stress $\left(42^{\circ} \mathrm{C}\right)$ and induced by low temperature $\left(4^{\circ} \mathrm{C}\right)$ treatment. Strong GUS staining was observed in the sites of meristematic tissues of pAtNRP1:: GUS transgenic plants, such as shoot apex and root tips, young leaf veins, stamens and stigmas of flowers, and abscission layers of young siliques. To study AtNRP1 biological functions, we have characterized both loss-of-function T-DNA insertion and transgenic overexpression plants for AtNRP1 in Arabidopsis. The T-DNA insertion mutants displayed no obvious difference as compared to wild-type Arabidopsis under heat stress, but the significant enhanced susceptibility to heat stress was revealed in two independent AtNRP1-overexpressing transgenic lines. Further study found that the decreased thermtolerance in AtNRP1overexpressing lines accompanied significantly decreased accumulation of ABA after heat treatment, which was probably due to AtNRP1 playing a role in negative-feedback regulation of the ABA synthesis pathway. These results support the viewpoint that the application of $A B A$ inhibits nodulation and nodulin-related gene expression and threaten adverse ambient temperature can impact the nodulin-related gene expression.
\end{abstract}

\section{INTRODUCTION}

Plants are exposed to various environmental stresses during their growth and development. Among these stresses, high temperature is one of the major problems that limit the growth and distribution of plants (Boyer, 1982). On the other hand, plants have evolved various mechanisms for adapting to the effects of heat shock (HS). Accumulation of heat shock proteins (HSPs), membrane compositional changes necessary for maintenance of functional integrity, and activation of oxidative defensive systems are involved in improving plant thermotolerance (Kaplan et al., 2004; Kotak et al., 2007b; Locato et al., 2008; Queitsch et al., 2000; Sung et al., 2001). Transcription activation of HSPs genes is regulated by heat shock transcrip- tion factors (HSFs) through binding to heat shock promoter elements (HSEs) in the promoter regions of HSPs genes during heat stress (Baniwal et al., 2004; Yamamoto et al., 2005). In addition, the induction of abscisic acid (ABA), salicylic acid (SA), and calcium-based signaling pathways were reported to be involved in heat-stress adaptation (Clarke et al., 2004; Larkindale and Knight, 2002; Larkindale et al., 2005; Liu et al., 2008). Exogenous application of these signaling agents to plants can also result in some degree of enhanced thermotolerance (Larkindale and Knight, 2002). These multiple responses suggest that many alternative processes are involved in thermotolerance. Plants at various growth stages respond differently to heat stress, suggesting a link between development and thermotolerance (Hong et al., 2003).

The infection of the plant by rhizobia bacteria is a complex process which a number of plant genes take part in. Legumes form a specialized organ termed the nodule via the expression of their genes that encode proteins named 'nodulins' when the leguminous plants were elicited by the secretion of bacterial effectors called nod factors in their root hairs (Stougaard, 2000). For non-nodule plants, Arabidopsis, when the plant roots have been colonized by several specific strains of Pseudomonas spp., they would develop a protective defense response that is called rhizobacteria-mediated induced systemic resistance (Cartieaux et al., 2003). Recent study has indicated that abscisic acid (ABA) can not only inhibit Nod factor signal transduction and promote or suppress resistance against various pathogens (de Torres-Zabala et al., 2007; Ding et al., 2008; Mohr and Cahill, 2007), but also can break a new signaling pathway to heat stress (Larkindale and Huang, 2004; Larkindale et al., 2005). A transient peak in ABA levels was reported in response to HS in pea plants (Liu et al., 2006) and during recovery from $\mathrm{HS}$ treatments in creeping bentgrass (Larkindale and Huang, 2004). It has been reported that ABA induces thermotolerance in cell-suspension cultures of Bromus inermis Leyss. (Robertson et al., 1994), and ABA pretreatment can increase cell viability and growth upon HS (Zhang and Fevereiro, 2007). In addition, the overexpression of transcription factor $\mathrm{ABF} 3$ in $\mathrm{ABA}$ signaling induced high-temperature tolerance in transgenic Arabidopsis (Kim et al., 2004). These results suggested that $A B A$ could also be involved in the response to heat stress. Moreover, genotypes with a putative high ABA

\footnotetext{
${ }^{1}$ Key Laboratory of Tropical Forest Ecology, Xishuangbanna Tropical Botanical Garden, Chinese Academy of Sciences, Kunming 650223, People's Republic of China, ${ }^{2}$ Graduate School of the Chinese Academy of Sciences, Beijing 100039, People's Republic of China

*Correspondence: ydq@xtbg.ac.cn
} 
level may be more tolerant to stresses (Xiong and Zhu, 2003). The Arabidopsis ABA signaling mutants abscisic acid insensitive 1 (abi1) and abi2 showed reduced survival after $\mathrm{HS}$, but the accumulation of HSPs was not affected in these mutants, and ABA does not appear to be required for HSP synthesis during HS (Larkindale et al., 2005). So it seems that ABA related heat tolerance may be independent on heat shock proteins synthesis pathwlay.

Nodulin-related protein 1, encoded by At2g03440, is a gene of unknown function encoding a putative mature protein of 187 amino acids. Bioinformatics analysis revealed that the amino acid sequence of the nodulin-related protein has some similarity to early nodulin 12B precursor (Bauer, 1994), and there are $41.3 \%$ identity $(63.0 \%$ similarity) in proline-rich domain of the nodulinrelated protein 1 and alfalfa early nodulin 12B precursor (see Supplementary Fig. 1), we designated the protein 'AtNRP1'. It has been demonstrated that AtNRP1 was an especially strong interactor of RPS2 (recognition of $P$. syringae strains) in yeasttwo-hybrid assay (Quirino et al., 2004), and a recent study showed that AtNRP1 was strongly up-regulated in Capsicum annuum hypersensitive induced reaction gene (CaHIR1)overexpressing transgenic Arabidopsis, and was also differentially induced at the transcriptional level by infection of $P$ seudomonas syringae pv. tomato DC3000 (Jung et al., 2008). However, the specific roles of AtNRP1 in Arabidopsis have not been determined so far.

In this study, we cloned AtNRP1 cDNA using a temperature screen. AtNRP1 expression was significantly suppressed by heat stress and induced by low temperature treatment. Histochemical analysis in pAtNRP1::GUS transgenic plants showed that AtNRP1 expressesed mainly in the apical meristematic tissues. The AtNRP1-overexpressing transgenic plants showed decreased thermotolerance, but the expression of some known heat-induced genes, such as HsfA2, MBF1c, Hsp101 and Hsp70, had no significant difference compared with wild-type plants under heat stress. Further study found that AtNRP1 was an ABA regulated gene, and the $\mathrm{ABA}$ accumulation in AtNRP1-overexpressing transgenic plants was lower than that of WT and nrp1 under heat stress. These results showed AtNRP1 might play a role in thermotolerance through the ABA synthesis pathway.

\section{MATERIALS AND METHODS}

\section{Plant materials and growth conditions}

The Arabidopsis thaliana ecotype Columbia was used as the WT for experiments in this study. The Arabidopsis seeds (WT, $n r p 1$, and AtNRP1-overexpressing transgenic plants) were surface sterilized for $15 \mathrm{~min}$ in $20 \%(\mathrm{v} / \mathrm{v})$ bleach, and rinsed twice with sterile distilled water. Seeds were grown on 1/2 Murashige and Skoog (MS) solid medium with $0.7 \%(\mathrm{w} / \mathrm{v})$ agar and $1.5 \%$ sucrose. After 3 days stratification in darkness at $4^{\circ} \mathrm{C}$, seeds were transferred to plant incubators with a $12 \mathrm{~h}$-light (100 $\left.\mu \mathrm{E} \mathrm{m}^{-2} \mathrm{~s}^{-1}\right) / 12 \mathrm{~h}$-dark photoperiod at $22^{\circ} \mathrm{C}$. The seedlings were either grown in axenic culture on $1 / 2 \mathrm{MS}$ medium or transferred into soil in the greenhouse $\left(22^{\circ} \mathrm{C}\right.$ with a $12 \mathrm{~h}$ photoperiod) $7 \mathrm{~d}$ after germination on $1 / 2 \mathrm{MS}$ medium.

\section{Isolation of T-DNA insertion mutants}

The AtNRP1 T-DNA insertion line (SALK_111768), in the Col background, was obtained from the Arabidopsis Biological Resource Center (ABRC; Ohio State University, USA). The TDNA insertion sites were confirmed by PCR using a T-DNA border primer (5'-AAACGTCCGCAATGTGTTAT-3') and the gene-specific primer (5'-ACGAGTCAGACAAGCTTGACA-3'). Plants homozygous for the T-DNA insertion were identified by another PCR using the above gene-specific primer and genespecific reverse primer (5'-TTTGCCAAAACCTAAAAATTATCA $-3^{\prime}$ ) and Northern blot analysis.

Construction of AtNRP1-overexpressing transgenic plants The full-length cDNA of AtNRP1 (U22696) was obtained from $\mathrm{ABRC}$ and confirmed by sequence analysis. To generate the 35S::AtNRP1 construction, the full-length cDNA fragment of AtNRP1 was cloned into the BamHI and Sall sites of pOCA30 (Chen and Chen, 2002). The recombinant plasmid was introduced into Agrobacterium tumefaciens strain GV3101 and followed by transformation into wild-type Arabidopsis plants by the floral dip method (Clough and Bent, 1998). The seeds were collected from the $A$. tumefaciens-infected plants and selected on $1 / 2 \mathrm{MS}$ agar medium containing $50 \mathrm{\mu g} \mathrm{ml}^{-1}$ kanamycin. Northern blot analysis was performed to further identify transgenic plants. $\mathrm{T}_{3}$ homozygous AtNRP1-overexpressing transgenic plants were selected for further experiments.

\section{Heat treatments assays}

Thermotolerance assays of seven-day-old normal-grown and 2.5-day-old dark-grown seedlings were performed as described by Larkindale et al. (2005) with minor modifications. Seven-dayold seedlings were exposed to a $45^{\circ} \mathrm{C} \mathrm{HS}$ for 260 min after a pretreatment for $90 \mathrm{~min}$ at $38^{\circ} \mathrm{C}$ and $120 \mathrm{~min}$ at $22^{\circ} \mathrm{C}$, and then returned to $22^{\circ} \mathrm{C}$ to recover for $7 \mathrm{~d}$. Seedlings were photographed to record viability, and the seedlings that were still green and producing new leaves were scored as surviving. The survival rate was the ratio of surviving seedlings to total treated seedlings. For the hypocotyl elongation assay, 2.5-day-old dark-grown seedlings on vertical plates were heat-treated at $45^{\circ} \mathrm{C}$ for 180 or $220 \mathrm{~min}$ after a pretreatment for $90 \mathrm{~min}$ at $38^{\circ} \mathrm{C}$ and $120 \mathrm{~min}$ at $22^{\circ} \mathrm{C}$. After an additional 2.5 days growing in the dark, seedlings were measured and photographed. In these assays, nrp1 and AtNRP1-overexpressing transgenic plants were compared with WT plants growing on the same plate to ensure the compared plants received identical stress conditions. Three-week-old WT, nrp1 and AtNRP1-overexpressing transgenic plants cultured in soil were heat treated at $42^{\circ} \mathrm{C}$ for $0 \mathrm{~min}$, $30 \mathrm{~min}$, or $90 \mathrm{~min}$ for northern blot analysis.

\section{Electrolyte leakage assays}

To explore the difference in thermotolerance of adult plants, leaves subjected to heat stress were assayed for electrolyte leakage according to methods of Clark et al. (2004). The plants were cultured in soil for 3 weeks, and the 3rd to 5th leaves were used for electrolyte leakage tests. The leaves were placed in glass tubes containing $10 \mathrm{ml}$ distilled water and were incubated in the water bath at $42^{\circ} \mathrm{C}$ in the dark. Water conductance was measured at intervals during heat treatment and calculated per milligram FW.

\section{Quantification of ABA content}

The ABA content was assayed using enzyme-linked immunosorbent assays (ELISA) as previously described (Yang et al., 2001) Three-week-old seedlings were treated at $38^{\circ} \mathrm{C}$ for $0.5,1$ and $2 \mathrm{~h}$. Approximately $0.5 \mathrm{~g}$ tissue samples were frozen in liquid nitrogen and ground to a fine powder. Endogenous $A B A$ was extracted and the content analyzed by ELISA.

\section{RNA isolation and gel blotting}

Total RNA was extracted from three-week-old Arabidopsis plants using the TRIZOL reagent (Invitrogen). For RNA gel blot analysis, total RNA $(10 \mu \mathrm{g})$ was separated on $1.2 \%$ agaroseformaldehyde gels and blotted onto nylon membranes. Blots 
A
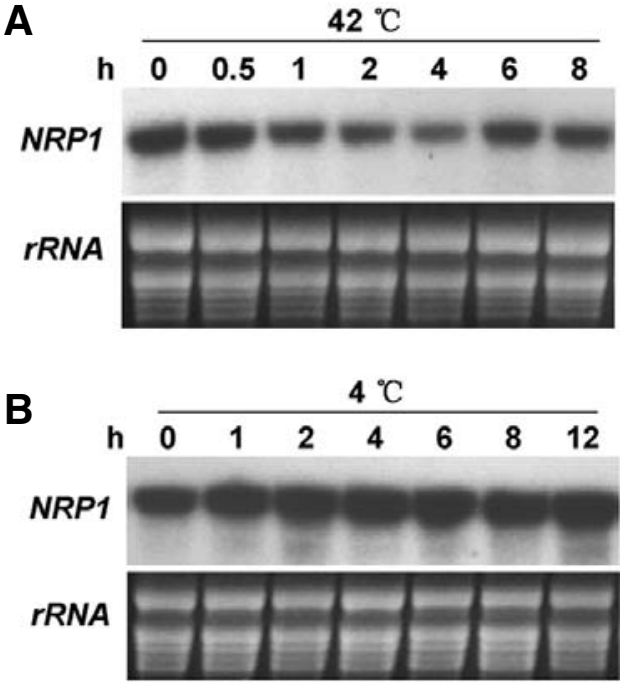

Fig. 1. Expression analysis of AtNRP1. Expression of AtNRP1 in response to (A) $42^{\circ} \mathrm{C}$ and $(B) 4^{\circ} \mathrm{C}$. Each lane was loaded with 10 $\mu \mathrm{g}$ total RNA prepared from 3-week-old plants treated with the various stresses as described in experimental procedures. Equal loadings were confirmed by Ribosomal RNA prestained gels with ethidium bromide. The 400 bp specific fragment of AtNRP1 cDNA was used for the probe. The experiments were repeated at least three times with similar results. $h$, hours post-treatment.

were hybridized with $\alpha-{ }^{32} \mathrm{P}-\mathrm{dATP}$-labeled gene-specific probes corresponding to the following genes: AtNRP1, HsfA2, MBF1c, $H s p 70$ and $H s p 101$, respectively, following standard procedures. Hybridization was performed in PerfectHyb plus hybridization buffer (Sigma-Aldrich) overnight at $68^{\circ} \mathrm{C}$. The membrane was then washed according to the procedure described by $\mathrm{Yu}$ et al. (2001).

\section{PAtNRP1::GUS construction and GUS staining}

To clone the promoter of AtNRP1, a 1866-bp AtNRP1 promoter region was amplified from Arabidopsis genomic DNA by PCR using the specific forward primer 5'-TTGCGGCCGCAATGAAAGATCCGGAAAGTGTAATTT-3' and the specific reverse primer 5'-TTTCTAGAGTTCTITGTTCAAAGGAAAAGTT-3', which contain a Notl and a Xbal restriction sites (underlined), respectively. The PCR fragment was digested with Notl and Xbal, and was firstly inserted in frame upstream of the glucuronidase (GUS) reporter gene in pJS131B vector. The recombinant plasmids were digested with Smal and $B a m H I$, and the resulting AtNRP1 promoter-GUS fusion fragments were cloned into transformation vector pOCA28 (Du and Chen, 2000).

GUS staining was performed using the method of Sieburth and Meyerowitz (1997). Leaves or tissues were gently fixed by incubation in $90 \%$ acetone on ice for $20 \mathrm{~min}$ and were then transferred into staining solution that contains $50 \mathrm{mM}$ sodium phosphate (pH 7.0), $0.5 \mathrm{mM} \mathrm{K}_{3} \mathrm{Fe}(\mathrm{CN})_{6}, 0.5 \mathrm{mM} \mathrm{K}_{4} \mathrm{Fe}(\mathrm{CN})_{6}, 0.1 \% \mathrm{v} / \mathrm{v}$ Triton X-100 and $2 \mathrm{mM} X$-gluc (Sigma-Aldrich), vacuum infiltrated for $5 \mathrm{~min}$, and incubated in darkness at $37^{\circ} \mathrm{C}$ overnight. After staining, tissues were washed with $70 \%$ ethanol.

\section{RESULTS}

\section{Expression level of AtNRP1 is regulated by temperature} stress treatments

In order to determine the biological function of AtNRP1, we inves- tigated its expression pattern in response to different stress with northern blot analysis. Three-week-old seedlings were treated with either $42^{\circ} \mathrm{C}, 4^{\circ} \mathrm{C}, 300 \mathrm{mM} \mathrm{NaCl}$ or natural drought at indicated time. As shown in Fig. 1, expression of the AtNRP1 gene was readily affected by temperature stress. The expression of AtNRP1 decreased significantly and a minimum expression level was reached at $4 \mathrm{~h}$ after $42^{\circ} \mathrm{C}$ treatment, followed by a gradual recovery of transcription to the background level at $6 \mathrm{~h}$ (Fig. 1A). On the other hand, AtNRP1 transcription was induced by lowtemperature stress. A rapid accumulation of AtNRP1 transcripts was observed $2 \mathrm{~h}$ after initiation of cold treatment, and these accumulated over a period of $12 \mathrm{~h}$ with a maximum level of transcripts attained after 4-6 hours (Fig. 1B). Expression of the AtNRP1 gene was down-regulated slightly under $\mathrm{NaCl}$ stress and did not respond to dehydration stress (data not shown). These results suggest that AtNRP1 is very sensitive to environmental stimuli, and it appears to be positively regulated by cold stress but negatively by heat stress. Collectively, these data indicate that AtNRP1 may behave as a stress-responsive gene.

\section{Developmental expression pattern directed by pAtNRP1::GUS fusion}

To study the expression pattern of AtNRP1 in different developmental stages and different tissues, we analyzed transgenic Arabidopsis plants expressing a $\beta$-glucuronidase (GUS) reporter gene driven by AtNRP1 promoter. GUS staining was observed in root tips of 1- and 2-day-old pAtNRP1::GUS transgenic seedlings after germination, whole plant of 3- and 5-dayold pAtNRP1::GUS transgenic seedlings grown in normal condition, root tips and junctions of roots and hypocotyls of 5-dayold transgenic seedlings grown in darkness (Figs. 2A-2E). With the rapid growth, GUS staining enhanced greatly in 10-day-old transgenic seedlings (Fig. 2F). While the expression of AtNRP1 dramatically declined with further growth of three-week-old transgenic seedlings, GUS staining was only found in vasculature, hydathodes of adult leaves, the sites of meristematic tissues, such as vegetative shoot apex and root tips (Fig. 2G). AtNRP1 also expressed in developing organs such as stamens and stigmas of flowers, and abscission layers of young siliques (Fig. $2 \mathrm{H}$ ). Interestingly, the expression level of the AtNRP1 gene in apical meristematic regions was much higher than that in other regions.

\section{Overexpression of AtNRP1 results in altered response to heat stress}

In order to further determine the function of AtNRP1, we identified a T-DNA insertion mutant for AtNRP1. The mutant (Salk 111768) contains a T-DNA insertion in the extron region of AtNRP1 (Fig. 3A), and the homozygous mutant plants were confirmed by PCR with gene-specific primers. Northern blot analysis used a DNA fragment that corresponds to the region downstream of the T-DNA insertion site in AtNRP1 as the probe. The AtNRP1 transcripts were detected in WT plants but not in nrp1 under normal conditions (Fig. 3B). Mutant plants grow and develop normally, with no obvious differences in morphological or growth phenotype from that of WT plants (data not shown).

To characterize the effect of AtNRP1 overexpression, Arabidopsis WT plants were transformed with a full-length AtNRP1 cDNA driven by the CaMV 35S promoter. Overexpression of AtNRP1 was confirmed by RNA gel blot analysis in the independent lines. Besides the level of AtNRP1 expression, no other notably different morphological or growth phenotypes were observed between WT plants and the 35S::AtNRP1 transgenic lines (data not shown). Two transgenic lines, line 3 and line 8, 
A

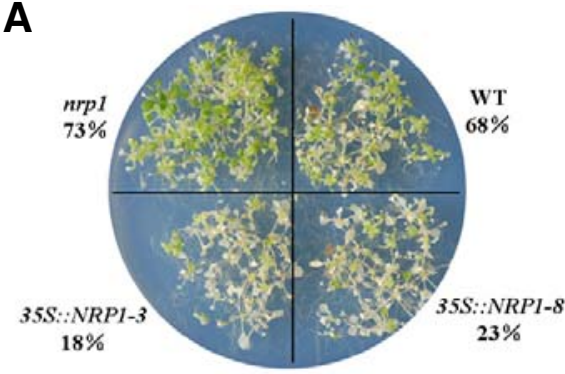

C

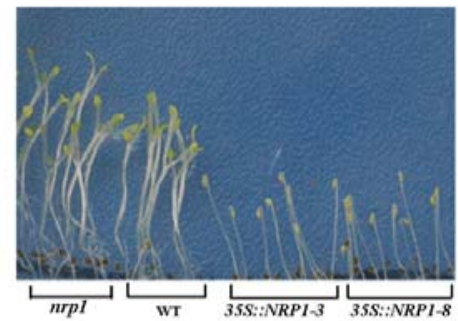

B

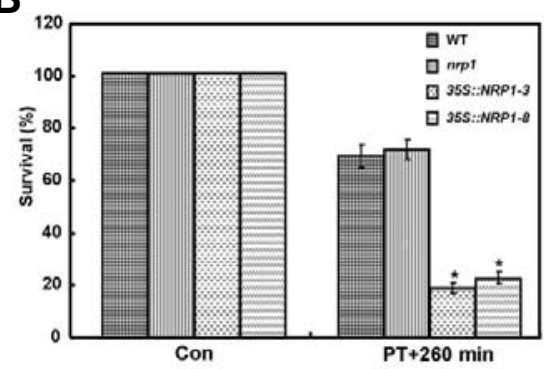

D

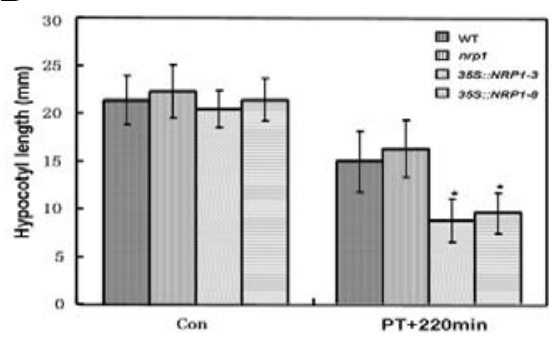

E

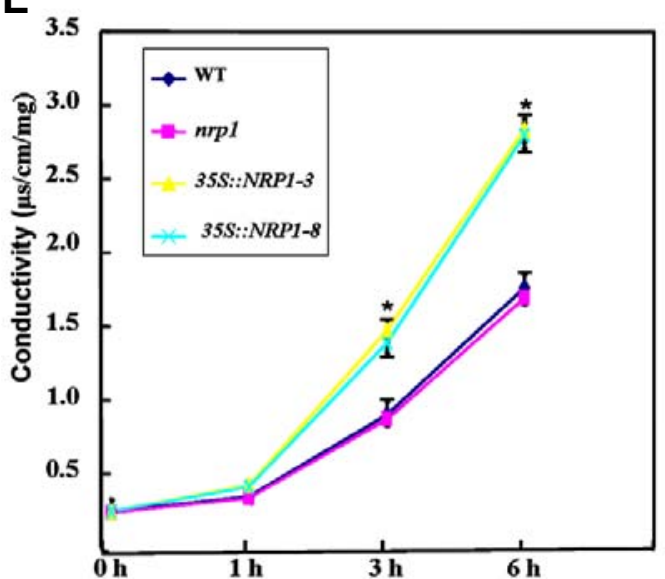

Fig. 4. Altered responses of 35S: AtNRP1 plants to heat stress. (A) Sevenday-old seedlings of wild-type, $n r p 1$ and 35S::AtNRP1 lines grown on 1/2 MS solid medium were treated at $45^{\circ} \mathrm{C}$ for $260 \mathrm{~min}$ after a pretreatment for $90 \mathrm{~min}$ at $38^{\circ} \mathrm{C}$ and $120 \mathrm{~min}$ at $22^{\circ} \mathrm{C}$. Seedlings were scored and photographed after recovery for an additional 7 days. (B) Percentage survival after heat treatment in the experiment summarized in (A). Data are presented as the mean \pm SD $(n=3)$. Each replicate consisted of 50 seedlings. Date were analyzed using the MannWhitney $U$ test; significant at ${ }^{*} P<0.05$. (C) Hypocotyl elongation of 2.5-day-old seedlings. Seedlings were grown on vertical plates in the dark for $2.5 \mathrm{~d}$ and, following a pretreatment for $90 \mathrm{~min}$ at $38^{\circ} \mathrm{C}$ and $120 \mathrm{~min}$ at room temperature, and $220 \mathrm{~min}$ heat stress at $45^{\circ} \mathrm{C}$, received a further $2.5 \mathrm{~d}$ recovery. (D) Hypocotyl length recorded after the final 2.5 days recovery in the experiment summarized in (C). Data are presented as the mean \pm SD $(n=3)$. Each replicate consisted of 40 seedlings. Data were analyzed using the Mann-Whitney $U$ test; significant at ${ }^{*} P<$ 0.05. (E) Electrolyte leakage assay performed on 21-day-old seedlings grown in soil. The $3^{\text {rd }}-5$ th leaves of WT, nrp 1 and AtNRP1-over-expressing lines were incubated in a $42^{\circ} \mathrm{C}$ water bath for 1,3 or $6 \mathrm{~h}$, and transferred to $22^{\circ} \mathrm{C}$, when the bathing solution conductivity was monitored. Data are presented as the means of three experiments each with 10 plants per treatment. Bars represent the SD $(n=$ 20). Data were analyzed using the MannWhitney $U$ test; significant at ${ }^{*} P<0.05$.

\section{ABA content analysis}

ABA can inhibit Nod factor signal transduction of resistance to various pathogens (de Torres-Zabala et al., 2007; Ding et al., 2008), and some studies have indicated that the ABA content was increased in response to heat stress (Kim et al., 2004; Zhang and Fevereiro, 2007). So we propose AtNRP1 may function in thermotolerance through the ABA signaling pathway. One assumption is that the decreased heat tolerance of AtNRP1-overexpressing lines under heat stress resulted from alteration of endogenous ABA homeostasis. Quantification of ABA content in AtNRP1-overexpressing lines, WT and nrp1 plants under heat stress showed that ABA content rapidly accumulated in all seedlings under heat stress after $30 \mathrm{~min}$, and increased continuously for the duration of the heat-stress treatment, however, AtNRP1-overexpressing transgenic lines accumulated substantially lower amounts of ABA than WT and nrp1 (Fig. 6A). We also analyzed AtNRP1 expression in response to ABA treatment. As shown in Fig. 6B, AtNRP1 expression decreased gradually after treatment with $100 \mu \mathrm{M}$ ABA. In contrast, no significant difference was observed in AtNRP1 expression after treatment with water control. Northern blot analysis of AtNRP1 expression in the ABA biosynthesis mutant aba2 under heat stress showed that heat-stress-induced re- pression of AtNRP1 transcription was abolished, and the aba2 have higher level of AtNRP1 compared to WT under heat stress (Fig. 6C). These data indicate that AtNRP1 gene may be regulated by the $A B A$ synthesis pathway during heat stress.

\section{DISCUSSION}

AtNRP1 was an interacting protein of RPS2, and took part in response in restricting growth of DC3000 (avrRpt2 ${ }^{+}$) (Quirino et al., 2004). AtNRP1 was a nodulin-related protein, and regulated by temperature stress and ABA treatment (Figs. 1 and 6B). AtNRP1 may play a role in tolerance to pathogens and the extreme temperatures.

In the present study, we analyzed preliminarily the expression profile and GUS staining-histochemical location of AtNRP1. Northern blot showed that AtNRP1 expression showed that AtNRP1 was suppressed by heat treatment, however rapidly increased during cold stress (Fig. 1), suggesting it was ready to be affected by adverse ambient temperature. Extreme temperature is one of major factor which affect plant growth and development, and many genes expression had similar patterns in response to temperature factor, that is to say, the genes are induced or suppressed by high or low temperature stress (Fu et al., 


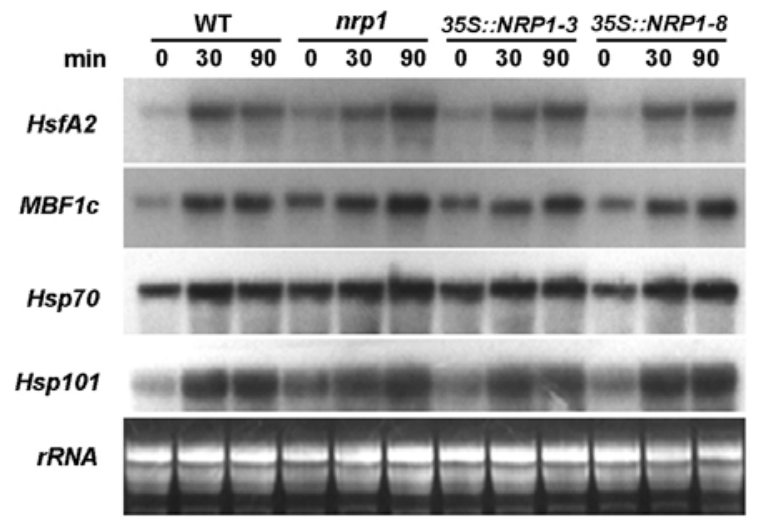

Fig. 5. Expression of heat-induced genes in three-week old WT, $n r p 1$ and 35S::AtNRP1 plants during heat treatment. Total RNA (10 $\mu \mathrm{g})$ was loaded into each lane and Ribosomal RNA was used as a control for RNA loading. The gene-specific fragments for HsfA2, MBF1c, Hsp70, and Hsp101 were used for the probe. The experiments were repeated three times with similar results.

1998; Knight and Knight, 2001), however, AtNRP1 was suppressed with heat treatment and induced during cold stress. Response mechanism of AtNRP1 in high and low temperature stress may be different. Further characterization is still needed to determine the specific functions of AtNRP1 in temperature stress. It is clear that genes affected by multiple types of stress are general and potentially confer tolerance to environmental stress (Aviezer- Hagai et al., 2007; Hazen et al., 2003). AtNRP1 expression is affected by temperature factor (Fig. 1), suggesting that AtNRP1 may play a role in resistance to environmental stress. GUS staining demonstrated that AtNRP1 expressed in the sites of meristematic tissues such as the vegetative shoot apex and root tips, developing organs such as floral buds, stamen and pistils of young flowers, abscission layers of immature siliques and junctions of pedicels (Fig. 2). Considering the specificity of the expression, AtNRP1 may play a certain function in development of Arabidopsis seedlings and floral organs. In addition, these tender tissues are sensitive to environmental factors and are readily to affect by stresses (Cazalé et al., 2009).

To elucidate the biological function of AtNRP1, AtNRP1 null mutants and AtNRP1-overexpressing transgenic plants were generated (Fig. 3). Transgenic plants overexpressing AtNRP1 exhibited less tolerance to high temperature stress than WT and nrp1. This reduced tolerance was illustrated by both the lower survival rate of seven-day-old seedlings and shorter hypocotyl elongation of 2.5-day-old dark-grown seedlings under heat stress (Figs. 4A and 4C). Electrolyte leakage assays also showed that the three-week-old overexpressing transgenic plants were thermosensitive compared with WT (Fig. 4E). Hypocotyl elongation, survival rate of seedling, and electrolyte leakage assay are effective parameters in analysis of heat tolerance (Clark et al., 2004; Hong and Vierling, 2000; Li et al., 2009). The AtNRP1-overexpressing transgenic plants showed decreased thermotolerance at different growth stages and the null mutation at the AtNRP1 gene was the same as WT under heat stress, which indicated that the single-gene mutation of AtNRP1 was insufficient to alter heat tolerance. There are many cases where a T-DNA KO line does not show any phenotype because of functional complementation of related genes (Cutler and McCourt, 2005; Higashi et al., 2008; Lee, et al., 2008). AtNRP1 may have functional overlap with other heat-related genes, and these functional-overlap genes may compensate for
A

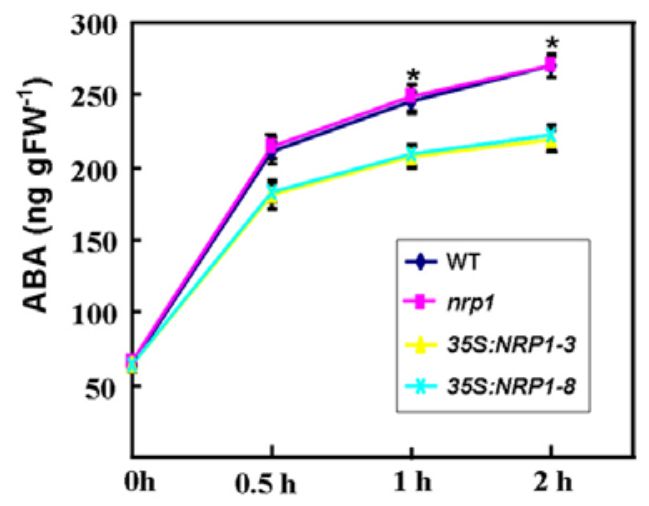

B

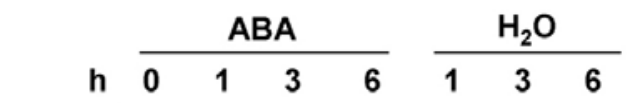

NRP1

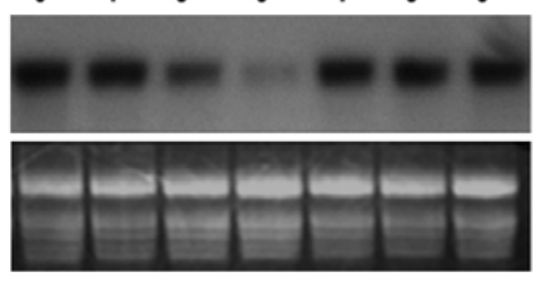

C

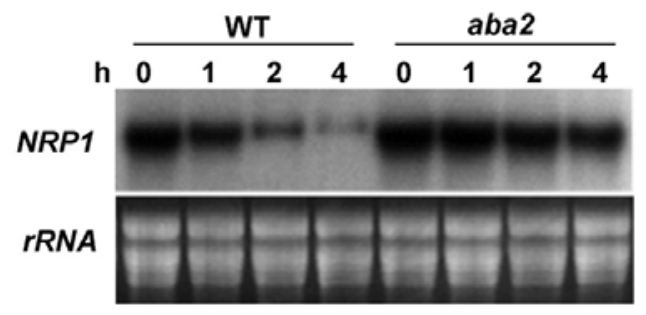

Fig. 6. Analysis of the molecular mechanisms of decreased thermotolerance in 35S::AtNRP1 plants. (A) ABA content in WT, nrp1, and 35S::AtNRP1 transgenic plants after heat treatment at $38^{\circ} \mathrm{C}$ for 0.5 , 1 or $2 \mathrm{~h}$. Three-week-old WT, nrp1, and 35S::AtNRP1 transgenic plants grown in soil were harvested after heat treatment for the indicated time periods. ABA content was determined by ELISA analysis. The results showed 35S::AtNRP1 overexpressing plants accumulated low levels of $A B A$ with three independent experiments in the same condition. Data are presented as the mean \pm SD $(n=$ 10). Date were analyzed using the Mann-Whitney $U$ test; significant at ${ }^{*} \mathrm{P}<0.05$. (B) Expression of AtNRP1 in response to application ABA. (C) Expression of AtNRP1 in WT and aba2-3 during heat treatment. Total RNA $(10 \mu \mathrm{g})$ was loaded into each lane and Ribosomal RNA was used as the control for RNA loading. The genespecific fragment of AtNRP1 was used for the probe. The experiments were repeated three times with similar results.

the lack of function of AtNRP1, so there are no obvious phenotype differences between $n r p 1$ and WT under nomal conditions or heat stress. Thus, we deduced that AtNRP1 might play a negative role in thermotolerance.

This decreased thermotolerance exhibited by AtNRP1overexpressing transgenic plants may be due to the decreased expression of heat-stress-inducible HSFs and HSPs genes. AtHsfA2 is an important regulator of thermotolerance, and is required for extension of acquired thermotolerance in Arabidopsis (Charng et al., 2007). AtHsp101 and AtHsp70 are believed to play a pivotal role in thermotolerance in Arabidopsis 
(Queitsch et al., 2000; Sung et al., 2001). The transcriptional coactivator MBF1c, is a key regulator of thermotolerance in Arabidopsis thaliana, and MBF1c is not required for the expression of transcripts encoding HsfA2 and different heat shock proteins in response to heat stress (Suzuki et al., 2008). Expression of HsfA2, MBF1c, Hsp70 and Hsp101 were characterized in among AtNRP1-overexpressing plants, $n r p 1$ and WT under heat stress. Northern blot analyses indicated that over-expressing of AtNRP1 did not alter the expression of these known heat inducible genes. Expression levels of HSFA2, MBF1c, HSP101 and HSP70 are same among the over-expression, nrp1 and WT under heat stress (Fig. 5). These results suggest that the heat sensitivity of AtNRP1-overexpressing plants is not conferred by these heat-induced genes, and other signaling pathways may be involved in the heat stress response of AtNRP1-overexpressing plants.

ABA acts through complex signalling networks to induce changes in multiple physiological processes. ABA levels increase when plants encounter adverse environmental conditions, and increased ABA content is beneficial for plants under environmental stress (Xiong and Zhu, 2003; Zhu, 2002). The accumulation of substantially amounts of ABA in nrp1 and WT plants was higher than in AtNRP1-overexpressing transgenic lines (Fig. 6A), which was consistent with their relative thermotolerance phenotypes. The signals of environmental stresses trigger ABA synthesis, which in turn induces expression of various responsive genes. These downstream genes also appear to involve extensive feedback regulation of $A B A$ accumulation (Uno et al., 2001; Verslues and Bray, 2006). The expression level of AtNRP1 was higher in aba2 than in wild-type under heat stress (Fig. 6C). Heat stress-induced inhibition of AtNRP1 expression is likely to be mediated through the ABA synthesis pathway, and AtNRP1 may affect feedback regulation of ABA accumulation in AtNRP1-overexpressing lines during heat stress. These results suggest that AtNRP1 takes part in heat stress response in plants via regulation of the $A B A$ synthesis pathway.

Although the molecular mechanisms underlying the roles of AtNRP1 under heat stress conditions are not clearly understood, the alteration of $\mathrm{ABA}$ accumulation in 35S::AtNRP1 plants compared with wild-type plants suggests a possible function of AtNRP1 under heat-stress conditions. It appears that the change in ABA accumulation in AtNRP1-overexpressing plants partly contributes to the decreased heat-stress resistance of AtNRP1 transgenic plants.

In conclusion, this paper presents these novel experimental data that increase our knowledge of the roles of AtNRP1 in response to environmental stresses. Phenotypic analysis of AtNRP1-overexpressing transgenic plants and knockout mutants implies that AtNRP1 may play a role as a negative regulator of seedling growth under heat stress. Whether the AtNRP1overexpressing plants and/or the loss-of-function mutant plants exhibit altered responses to other abiotic stress conditions has not been determined. Future studies in this direction may reveal not only additional roles for AtNRP1 in plant stress responses, but also possible interactions between their respective signaling pathways.

Note: Supplementary information is available on the Molecules and Cells website (www.molcells.org).

\section{ACKNOWLEDGMENTS}

We thank the ABRC at the Ohio State University (Columbus, $\mathrm{OH}$ ) for seeds of the Arabidopsis mutants. This work was supported by the Natural Science Foundation of China (grant no.
90817003), the Ministry of Science and Technology of China (grant no. 2006AA02Z129), and the Hundred Talents Program of the Chinese Academy of Sciences.

\section{REFERENCES}

Aviezer-Hagai, K., Skovorodnikova, J., Galigniana, M., FarchiPisanty, O., Maayan, E., Bocovza, S., Efrat, Y., von KoskullDöring, P., Ohad, N., and Breiman, A. (2007). Arabidopsis immunophilins ROF1 (AtFKBP62) and ROF2 (AtFKBP65) exhibit tissue specificity, are heat-stress induced, and bind HSP90. Plant Mol. Biol. 63, 237-255.

Baniwal, S.K., Bharti, K., Chan, K.Y., Ganguli, A., Kotak, S., Mishra, S.K., Port, M., Scharf, K.D., Tripp, J., Weber, C., et al. (2004). Heat stress response in plants: a complex game with chaperones and more than twenty heat stress transcription factors. J. Biosci. 29, 471-487

Bauer, P., Crespi, M.D., Szécsi, J., Allison, L.A., Schultze, M., Ratet, P., Kondorosi, E., and Kondorosi, A. (1994). Alfalfa Enodl2 genes are differentially regulated during nodule development by Nod factors and Rhizobium invasion. Plant Physiol. 105, 585592

Boyer, J.S. (1982). Plant productivity and environment. Science 218 443-448.

Cartieaux, F., Thibaud, M.C., Zimmerli, L., Lessard, P., Sarrobert, C., David, P., Gerbaud, A., Robaglia, C., Somerville, S., and Nussaume, L. (2003). Transcriptome analysis of Arabidopsis colonized by a plant-growth promoting rhizobacterium reveals a general effect on disease resistance. Plant J. 36, 177-188.

Cazalé, A.C., Clément, M., Chiarenza, S., Roncato, M.A., Pochon, N., Creff, A., Marin, E., Leonhardt, N., and Noël, L.D. (2009). Altered expression of cytosolic/nuclear HSC70-1 molecular chaperone affects development and abiotic stress tolerance in Arabidopsis thaliana. J. Exp. Biol. 60, 2653-2664.

Charng, Y.Y., Liu, H.C., Liu, N.Y., Chi, W.T., Wang, C.N., Chang, S.H., and Wang, T.T. (2007). A heat-inducible transcription factor, HsfA2, is required for extension of acquired thermotolerance in Arabidopsis. Plant Physiol. 143, 251-262.

Chen, C., and Chen, Z. (2002). Potentiation of developmentally regulated plant defense response by AtWRKY18, a pathogeninduced Arabidopsis transcription factor. Plant Physiol. 129, 706-716.

Clarke, S.M., Mur, L.A., Wood, J.E., and Scott, I.M. (2004). Salicylic acid dependent signaling promotes basal thermotolerance but is not essential for acquired thermotolerance in Arabidopsis thaliana. Plant J. 38, 432-447.

Clough, S.J., and Bent, A.F. (1998). Floral dip: a simplified method for Agrobacterium-mediated transformation of Arabidopsis thaliana. Plant J. 16, 735-743.

Cutler, S., and McCourt, P. (2005). Dude, where's my phenotype? dealing with redundancy in signaling networks. Plant Physiol. 138, 558-559.

de Torres-Zabala, M., Truman, W., Bennett, M.H., Lafforgue, G., Mansfield, J.W., Rodriguez Egea, P., Bögre, L., and Grant, M. (2007). Pseudomonas syringae pv. tomato hijacks the Arabidopsis abscisic acid signalling pathway to cause disease. EMBO J. 26, 1434-1443.

Ding, Y., Kalo, P., Yendrek, C., Sun, J., Liang, Y., Marsh, J.F., Harris, J.M., and Oldroyd, G.E. (2008). Abscisic acid coordinates nod factor and cytokinin signaling during the regulation of nodulation in Medicago truncatula. Plant Cell 20, 2681-2695.

Du, L., and Chen, Z. (2000). Identification of genes encoding novel receptor-like protein kinases as possible target genes of pathogen-induced WRKY DNA-binding proteins. Plant J. 24, 837-847.

Fu, P., Wilen, R.W., Robertson, A.J., Low, N.H., Tyler, R.T., and Gusta, L.V. (1998). Heat tolerance of cold acclimation puma winter rye seedlings and the effect of a heat shock on freeing tolerance. Plant Cell Physiol. 39, 942-949.

Hazen, S.P., Wu, Y., and Kreps, J.A. (2003). Gene expression profiling of plant responses to abiotic stress. Funct. Integr. Genomics 3, 105-111.

Higashi, K., Ishiga, Y., Inagaki, Y., Toyoda, K., Shiraishi, T., and Ichinose, Y. (2008). Modulation of defense signal transduction by flagellin-induced WRKY41 transcription factor in Arabidopsis thaliana. Mol. Genet. Genomics 279, 303-312.

Hong, S.W., and Vierling, E. (2000). Mutants of Arabidopsis thaliana. 
defective in the acquisition of tolerance to high temperature stress. Proc. Natl. Acad. Sci. USA 97, 4392-4397.

Hong, S.W., Lee, U., and Vierling, E. (2003). Arabidopsis hot mutants define multiple functions required for acclimation to high temperatures. Plant Physiol. 132, 757-767.

Jung, H.W., Lim, C.W., Lee, S.C., Choi, H.W., Hwang, C.H., and Hwang, B.K. (2008). Distinct roles of the pepper hypersensitive induced reaction protein gene CaHIR1 in disease and osmotic stress, as determined by comparative transcriptome and proteome analyses. Planta 227, 409-425.

Kaplan, F., Kopka, J., Haskell, D.W., Zhao, W., Schille, K.C., Gatzke, N., Sung, D.Y., and Guy, C.L. (2004). Exploring the temperature-stress metabolome of Arabidopsis. Plant Physiol. 136, 4159-4168.

Kim, J.B., Kang, J.Y., and Kim, S.Y. (2004). Over-expression of a transcription factor regulating ABA-responsive gene expression confers multiple stress tolerance. Plant Biotechnol. J. 2, 459-466.

Knight, H., and Knight, M.R. (2001). Abiotic stress signaling pathways: specificity and cross talk. Trends Plant Sci. 6, 262-267.

Kotak, S., Larkindale, J., Lee, U., van Koskull-Döring, P., Vierling, E., and Scharf, K.D. (2007). Complexity of the heat stress response in plants. Curr. Opin. Plant Biol. 10, 310-316.

Larkindale, J., and Knight, M.R. (2002). Protection against heat stress-induced oxidative damage in Arabidopsis involves calcium, abscisic acid, ethylene, and salicylic acid. Plant Physiol. 128, 682-695.

Larkindale, J., and Huang, B. (2004). Thermotolerance and antioxidant systems in Agrostis stolonifera: involvement of salicylic acid, abscisic acid, calcium, hydrogen peroxide, and ethylene. J. Plant Physiol. 161, 405-413.

Larkindale, J., Hall, J.D., Knight, M.R., and Vierling, E. (2005). Heat stress phenotypes of Arabidopsis mutants implicate multiple signaling pathways in the acquisition of thermotolerance. Plant Physiol. 138, 882-897.

Lee, S., Woo, Y.M., Rvu, S.I., Shin, Y.D., Kim, W.T., Park, K.Y., Lee, I.J., and An, G. (2008). Further Characterization of a Rice AGL12 Group MADS-Box Gene, OsMADS26. Plant Physiol. 147, 156-168.

Li, S., Fu, Q., Huang, W., and Yu, D. (2009). Functional analysis of an Arabidopsis transcription factor WRKY25 in heat stress. Plant Cell Rep. 28, 683-693.

Liu, H.T., Liu, Y.Y., Pan, Q.H., Yang, H.R., Zhan, J.C., and Huang, W.D. (2006). Novel interrelationship between salicylic acid, abscisic acid, and PIP2-specific phospholipase C in heat acclimation-induced thermotolerance in pea leaves. J. Exp. Bot. 57, 3337-3347.

Liu, H.T., Gao, F., Li, G.L., Han, J.L., Liu, D.L., Sun, D.Y., and Zhou, R.G. (2008). The calmodulin-binding protein kinase 3 is part of heat shock signal transduction in Arabidopsis thaliana. Plant $\mathrm{J}$. $55,760-773$.

Locato, V., Gadaleta, C., de Gara, L., and de Pinto, M.C. (2008). Production of reactive species and modulation of antioxidant network in response to heat shock: a critical balance for cell fate. Plant Cell Environ. 31, 1608-1619.
Mohr, P.G., and Cahill, D.M. (2007). Suppression by ABA of salicylic acid and lignin accumulation and the expression of multiple genes, in Arabidopsis infected with Pseudomonas syringae pv. tomato. Funct. Integr. Genomics 7, 181-191.

Queitsch, C., Hong, S.W., Vierling, E., and Lindquist, S. (2000). Heat shock protein 101 plays a crucial role in thermotolerance in Arabidopsis. Plant Cell 12, 479-492.

Quirino, B.F., Genger, R., Ham, J.H., Zabala, G., and Bent, A.F. (2004). Identification and functional analysis of Arabidopsis proteins that interact with resistance gene product RPS2 in yeast. Physiol. Mol. Plant. Pathol. 65, 257-267.

Robertson, A.J., Ishikawa, M., Gusta, L.V., and MacKenzie, S.L. (1994). Abscisic acid induced heat tolerance in Bromus inermis Leyss. cell-suspension cultures. Plant Physiol.105, 181-190.

Sieburth, L.E., and Meyerowitz, E.M. (1997). Molecular dissection of the AGAMOUS control region shows that cis elements for spatial regulation are located intragenically. Plant Cell 9, 355365.

Stougaard, J. (2000). Regulators and regulation of legume root nodule development. Plant Physiol. 124, 531-540.

Sung, D.Y., Vierling, E., and Guy, C.L. (2001). Comprehensive expression profile analysis of the Arabidopsis Hsp70 gene family. Plant Physiol. 126, 789-800.

Suzuki, N., Bajad, S., Shuman, J., Shulaev, V., and Mittler, R. (2008). The transcriptional co-activator MBF1c is a key regulator of the thermotolerance in Arabidopsis thaliana. J. Biol. Chem. 283, 9269-9275.

Uno, Y., Furihata, T., Abe, H., Yoshida, R., Shinozaki, K., and Yamaguchi-Shinozaki, K. (2000). Arabidopsis basic leucine zipper transcription factors involved in an abscisic acid-dependent signal transduction pathway under drought and high-salinity conditions. Proc. Natl. Acad. Sci. USA 97, 11632-11637.

Verslues, P.E., and Bray, E.A. (2006). Role of abscisic acid (ABA) and Arabidopsis thaliana. ABA-insensitive loci in low water potential-induced ABA and proline accumulation. J. Exp. Bot. 57, 201-212.

Xiong, L., and Zhu, J.K. (2003). Regulation of abscisic acid biosynthesis. Plant Physiol. 133, 29-36.

Yamamoto, A., Mizukami, Y., and Sakurai, H. (2005). Identification of a novel class of target genes and a novel type of binding sequence of heat shock transcription factor in Saccharomyces cerevisiae. J. Biol. Chem. 280, 11911-11919.

Yang, J., Zhang, J., Wang, Z., Zhu, Q., and Wang, W. (2001). Hormonal changes in the grains of rice subjected to water stress during grain filling. Plant Physiol. 127, 315-323.

Yu, D.Q., Chen, C., and Chen, Z.X. (2001). Evidence for an important role of WRKY DNA binding proteins in the regulation of NPR1 gene expression. Plant Cell 13, 1527-1540.

Zhang, C., and Fevereiro, P.S. (2007). The effect of heat shock on paclitaxel production in Taxus yunnanensis cell suspension cultures: role of abscisic acid pretreatment. Biotechnol. Bioeng. 96, 506-514.

Zhu, J.K. (2002). Salt and drought stress signal transduction in plants. Annu. Rev. Plant Physiol. 53, 247-273. 